\title{
Comparison of $3 T$ MR scanners in regional cartilage-thickness analysis in osteoarthritis: a cross-sectional multicenter, multivendor study
}

Sharon Balamoody ${ }^{1 *}$, Tomos G Williams ${ }^{1}$, John C Waterton ${ }^{1,2}$, Michael Bowes ${ }^{3}$, Richard Hodgson ${ }^{4}$, Chris J Taylor ${ }^{1}$, Charles E Hutchinson ${ }^{1,5}$

\begin{abstract}
Introduction: Cartilage thickness from MR images has been identified as a possible biomarker in knee osteoarthritis $(\mathrm{OA})$ research. The ability to acquire MR data at multiple centers by using different vendors' scanners would facilitate patient recruitment and shorten the duration of OA trials. Several vendors manufacture 3T MR scanners, including Siemens, Philips Medical Systems, and GE Healthcare. This study investigates whether quantitative MR assessments of cartilage morphology are comparable between scanners of three different vendors.

Methods: Twelve subjects with symptoms of knee OA and one or more risk factors had their symptomatic knee scanned on each of the three vendor's scanners located in three sites in the UK: Manchester (Philips), York (GE), and Liverpool (Siemens). The NIH OAl study protocol was used for the Siemens scanner, and equivalent protocols were developed for the Philips and GE scanners with vendors' advice. Cartilage was segmented manually from sagittal 3D images. By using recently described techniques for Anatomically Corresponded Regional Analysis of Cartilage (ACRAC), a statistical model was used anatomically to align all the images and to produce detailed maps of mean differences in cartilage-thickness measures between scanners. Measures of cartilage mean thickness were computed in anatomically equivalent regions for each subject and scanner image.
\end{abstract}

Results: The ranges of mean cartilage-thickness measures for this cohort were similar for all regions and across all scanners. Philips intrascanner root-mean-square coefficients of variation were low in the range from $2.6 \%$ to $4.6 \%$. No significant differences were found for thickness measures of the weight-bearing femorotibial regions from the Philips and Siemens images except for the central medial femur compartment $(P=0.04)$. Compared with the other two scanners, the GE scanner provided consistently lower mean thickness measures in the central femoral regions (mean difference, $-0.16 \mathrm{~mm}$ ) and higher measures in the tibial compartments (mean difference, $+0.19 \mathrm{~mm}$ ).

Conclusions: The OAl knee-imaging protocol, developed on the Siemens platform, can be applied to research and trials by using other vendors' $3 T$ scanners giving comparable morphologic results. Accurate sequence optimization, differences in image postprocessing, and extremity coil type are critical factors for interscanner precision of quantitative analysis of cartilage morphology. It is still recommended that longitudinal observations on individuals should be performed on the same scanner and that assessment of intra- and interscanner precision errors is undertaken before commencement of the main study.

\footnotetext{
* Correspondence: sharana@doctors.org.uk

'Biomedical Imaging Institute, The University of Manchester, Oxford Road,

Manchester, M13 9PT, UK

Full list of author information is available at the end of the article
} 


\section{Introduction}

Osteoarthritis (OA) is a major and increasing public health problem in the developed world. The present National Institutes of Health Osteoarthritis Initiative (NIH OAI) is a prospective ongoing 9-year study of approximately 5,000 subjects, investigating subjects both with $\mathrm{OA}$ and at risk of developing $\mathrm{OA}$ to identify measures of $\mathrm{OA}$ initiation and progression [1]. Cartilage thickness and volume measurements from MR images are potential morphologic biomarkers for OA that can be assessed from MR images, acquired annually on all subjects in the OAI. Such measures could be used as response biomarkers in the development of therapies for OA, particularly in Phase II clinical trials. All MR images in the OAI are acquired on four Siemens 3.0T MR scanners.

Recent analyses of small initial OAI cohorts suggest cartilage loss in OA to be in the range of $0.5 \%$ to $2.0 \%$ per annum [2]. Obesity, malalignment, and radiographic OA Kellgren and Lawrence grades 2 to 3 predispose to higher rates of cartilage loss [2-4]. Several factors can influence reproducibility of quantitative measurement of cartilage thickness between groups (for example, age and sex) and within groups (for example, recent weight-bearing activity, diurnal variation $[5,6])$. Phase II clinical studies of cartilage loss in OA are likely to require a multicenter approach to recruit sufficient numbers of subjects in a reasonable time with adequate statistical power. Most MR scanners are produced by one of three main vendors: Philips (Eindhoven, The Netherlands), Siemens (Erlangen, Germany), and GE Healthcare (Milwaukee, Wisconsin, USA). Multicenter, multivendor clinical studies of OA performed at 3.0T have been published [7]. Although evidence suggests that interscanner differences in cartilage volume in healthy subjects are small [8], little evidence indicates that quantitative analyses of degenerating cartilage on scanners of different vendors are comparable in symptomatic subjects and at higher field strength $[9,10]$.

The study described is a cross-sectional comparison of three 3.0T MR scanners of different manufacturers in the assessment of OA of the knee in a cohort of subjects with symptoms and risk factors for OA. The 3.0T scanners used are manufactured by Philips, Siemens, and GE. The imaging protocols are based on the Siemens NIH OAI protocol, which assesses several features of OA. A quantitative cartilage-morphology analysis is presented. The objectives were to quantify the interscanner precision errors and to assess whether these were significant by comparing with the intrascanner variability (assessed on the Philips scanner).

\section{Materials and methods Subjects}

Participants were recruited from the University of Manchester employee base by a universal email sent to all employees. Interested responders were subsequently sent an information sheet and a screening questionnaire. The questionnaire was designed to include contact information, demographics, and assessment of inclusion and exclusion criteria, which were based on those quoted in the NIH OAI protocol [11]. Subjects were required to have one or more symptoms of OA or one or more risk factors for OA or both. OA symptom criteria included pain, stiffness, locking (or requiring analgesia for symptoms) within the last month. Riskfactor criteria were obesity, family history of osteoarthritis, previous knee injury, and frequent knee-bending activity. Responders were excluded if they indicated definite or suspected history of inflammatory arthropathy or had contraindications to MR scanning. Suitable responders were invited for an MR scan. Informed consent was obtained from each participant. Ethical approval for the study was obtained from West Midlands Multi-Centre Research Ethics Committee (06/MRE07/16).

Seventeen subjects (12 men, five women) were initially recruited. Five of these subjects did not complete imaging at all three sites. Two of these five attended but failed to be scanned at one of the sites because of inadequate knee RF coil diameter, and therefore discontinued the study at that point. The other three subjects chose to withdraw before attending all three sites. Data acquired if subjects had not attended one or two of the sites was excluded from analysis, and therefore, the data for the 12 subjects who had been scanned at all three sites were analyzed. Demographics for this cohort $n=12$ as follows: nine men, three women, aged $49.3 \pm 10.0,32$ to 58 years (mean \pm standard deviation, range) and BMI $28.3 \pm 6.2$, 22.1 to $44.2 \mathrm{~kg} / \mathrm{m}^{2}$. Five of 12 subjects had knee pain on activity or rest or both for at least 7 days in the last month at the time of completing the questionnaire; the other seven subjects experienced pain less frequently. Three subjects previously had received a diagnosis of OA.

Although radiographic data were not available to demonstrate the presence of definite OA features, such as joint-space loss and osteophytes, for volunteer selection, as in the NIH study, semiquantitative knee scoring by using Whole-Organ Magnetic Resonance Score (WORMS) [12] was performed by a single observer (SB) on the acquired MR data. The WORMS involves dividing the total knee cartilage into 14 subregions (4 points for the patellofemoral (PF) joint; 5, for medial tibiofemoral (MTF) joint; and 5 for lateral tibiofemoral (LTF) joint). The cartilage in each subregion is scored from 0 to 6 ; $0=$ normal cartilage; $6=$ diffuse full-thickness cartilage loss. Then the relevant subregions are summed to obtain compartmental scores. With WORMS, maximal compartmental values obtainable would be 30,30 , and 12 for the MTF, LTF, and PF joints, respectively. 


\begin{tabular}{|c|c|c|c|}
\hline Vendor & Siemens & Philips & GE \\
\hline Sequence & 3D DESS & 3DWATSf & 3D GE \\
\hline $\begin{array}{l}\text { Fat saturation/water } \\
\text { excitation (WE) }\end{array}$ & WE & WE & Fat sat \\
\hline FOV $(\mathrm{mm})$ & 140 & 140 & 140 \\
\hline Number of slices & 160 & 120 & 210 \\
\hline Slice thickness (mm) & 0.7 & 0.7 & 1 \\
\hline Slice gap (mm) & 0 & 0 & -0.5 \\
\hline Flip angle (deg) & 25 & 25 & 25 \\
\hline $\mathrm{TE}(\mathrm{ms})$ & 4.7 & 9.2 & 4.9 \\
\hline TR (ms) & 16.3 & 20 & 16.8 \\
\hline X-resolution (mm) & 0.37 & 0.36 & 0.55 \\
\hline Y-resolution (mm) & 0.46 & 0.48 & 0.62 \\
\hline Scan time & $10 \min 35 \mathrm{~s}$ & $3 \min 51 \mathrm{~s}$ & $6 \mathrm{~min} 22 \mathrm{~s}$ \\
\hline
\end{tabular}

D.ESS, dual-echo steady state; GE, gradient echo; WATSf, water selective (fluid).

\section{The scanners}

The Siemens sequences were based on the NIH OAI protocol [11], whereas optimized protocols were obtained for the Philips and GE scanners. This was done with the help of clinical scientists specializing in musculoskeletal MR from Philips (Netherlands) and GE (UK). A summary of Siemens 3D DESS-we, and equivalent sequence parameters on the other scanners is given in Table 1. A notable difference is apparent with the GE sequence, which uses fat saturation in place of waterexcitation and also has a lower acquired resolution. Philips uses a higher TE/TR compared with the other two scanners $(9 / 20 \mathrm{~ms}$ versus $4 / 16 \mathrm{~ms})$.

The three 3.0T MR scanners were located as follows: Philips Achieva (Manchester, UK), Siemens Trio (MARIARC, Liverpool, UK), and GE Signa (YNIC, York, UK). The Siemens and GE coils used were quadrature circularly polarized transmit-receive and single-channel transmitreceive multipurpose HD knee/foot coil, respectively. An eight-channel SENSE phased-array receive coil was used at the Philips site. The SENSE function on the Philips coil was implemented for certain sequences only.

\section{Data acquisition}

Each subject was designated to have the same knee examined on each of the three different vendors, and, at the Philips 3.0T scanner, an additional examination at the same visit. Before scanning at each center, each subject underwent a minimum rest period of non-weight bearing of at least 30 minutes to minimize variability in cartilage thickness between scans due to recent weightbearing activity. It was not possible totally to control for within-subject diurnal variation in cartilage thickness between scans because of constraints on scanner and subject availability. Scan times for the OAI protocol (which comprised five acquisition sequences imaging the knee) at the Siemens, Philips, and GE scanners were 37 minutes 41 seconds, 39 minutes 9 seconds, and 41 minutes, respectively. At the Philips site, the sagittal 3D WATSf was acquired twice within the same session to assess intrascanner precision. After the first acquisition, the subject was removed from the magnet bore and allowed to sit on the scan table without weight bearing for approximately 10 to 15 minutes and repositioned for the second acquisition.

Efforts were made to ensure similar orientation of slices between scanners as follows. The planning for the first sagittal and coronal sequences was applied to subsequent sequences for each scan at each center. After the first of the three scans, the overlays of the scout views for sagittal and coronal images were saved and used for comparison for planning subsequent scans of the same subject on the other two scanners. Images at each of the sites were labeled with a unique study number randomized independently at each site so that observers would be blind to subject identity for each of the scanners. Blinding to scanner was not performed, as scanners could be easily identified from the images by their appearance.

\section{Quality assurance}

The routine quality assurance (QA) at each scanner site was recorded as follows. At the GE site, magnet SNR checks were performed on alternate days. No other documented quality control was available.

At the Siemens site, monthly/2-monthly QA reports were documented, including SNR, gradient sensitivity, and fat/water saturation checks. Some of the QA for the Siemens site was performed remotely.

At the Philips site, daily checks were performed by the radiographers. A dedicated employee responsible for scanner QA was available to perform checks by using phantoms investigating geometric distortion, SNR, RF uniformity, and slice profile. Most of the tests were stable. Regarding geometric distortion, slight drift was observed in the craniocaudal direction $(-0.1 \%$ to $0.2 \%)$ over the time course of the study. RF coil SNR checks were performed only occasionally at all sites.

\section{Cartilage segmentation}

In total, 48 scans were included in the cartilage morphology analysis: 12 subjects $\times$ four scans (three sites + extra 3D WATSf dataset acquired at Philips scanner). The cartilage was manually segmented from the sagittal $3 \mathrm{D}$ sequences by a single observer (author 1 ) blinded to subject identity, by using the Endpoint segmentation software (Imorphics, Manchester, UK), which implements a livewire algorithm [13]. Quality control of all 
segmentations was conducted by an experienced consultant musculoskeletal radiologist (last author). Closedsurface representations of the cartilage sheets were generated from the segmentations to enable volume and thickness to be measured in 3D.

\section{Defining a common frame of reference: bone reference surfaces}

To compare measurements of cartilage thickness from images of the same subject taken with different scanners, and to enable aggregation of measures over the study cohort, an anatomic frame of reference was defined in each image by using recently described techniques for Anatomically Corresponded Regional Analysis of Cartilage (ACRAC) [14]. The exosteal bone surface was chosen as a basis for the frame of reference because of the relative ease of its identification in the images and consistent topology across subjects. Six regions were defined on this surface; these were used for the analysis (see Figure 1). Statistical models were used as a means of defining the bone reference surface in each image [15]. The models provide a mean representation of all the training-set images and a warp from the mean image to each example image. When presented with new MR images, which were not part of the original training set, statistical models can also automatically find the best warp field from the mean image.

A statistical model was built from the DESS-we images of both knees fromthe 160 participants initially released by the OAI as group 0.B.1, which included a single time point only and both knees of participants [1]. The exosteal bone boundary was segmented by a consultant musculoskeletal radiologist (last author) in the mean model image, and a surface representation was generated. The warp fields from the mean image to the study images were found by the model and used to propagate the bone reference surface, thus providing anatomically coherent bone segmentations for each scan.

\section{Cartilage morphology measures}

The bone reference surfaces provided a dense set of points with mean separation of $0.6 \mathrm{~mm}$ on the distal femur and proximal tibia. Cartilage thickness was measured above each point in each image as the distance between the points of intersection of the 3D line, normal to the bone reference surface, with the inner and outer cartilage surfaces. The result is a cartilage-thickness measurement associated with each measurement point, which was displayed as a color-coded map of cartilage thickness on each reference bone surface.

From the individual cartilage-thickness maps, summary measures of cartilage morphology measures within anatomically consistent regions were computed to enable us to perform statistical analysis of the differences. From the bone reference surface, regions of interest, consistent with those proposed by Eckstein et al. [16] were delineated on the mean distal femoral and proximal tibial bone surfaces. Mean cartilage thickness over total bone area (ThCtAB, as defined by Eckstein et al. [16]), was computed for all regions as the average of the point-wise thickness measurements weighted by the area surrounding each measurement point. These regions by definition do not exclude any areas denuded of cartilage. Peripheral cartilage is more prone to segmentation error due to partial volume effect, as the

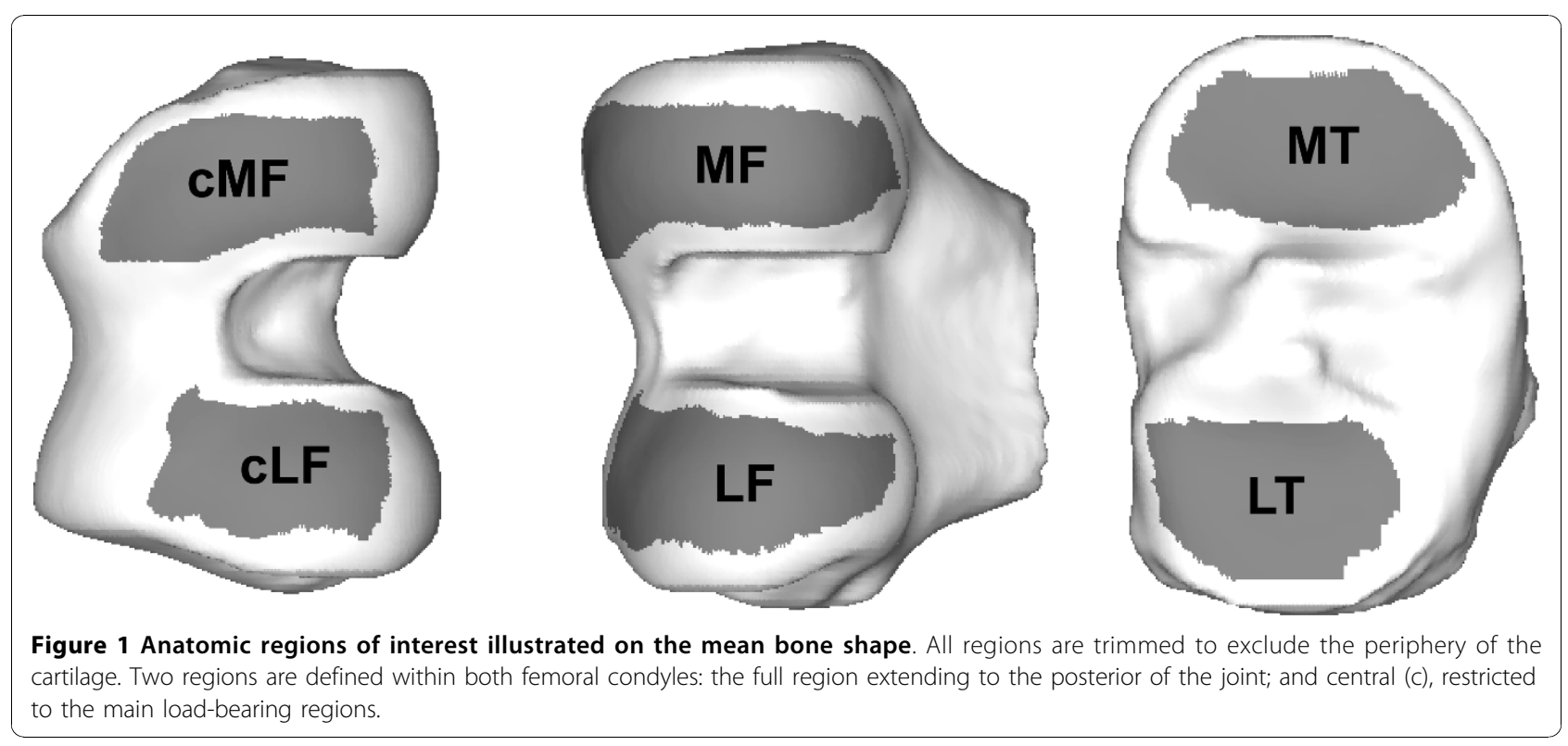


surface curves into the image plane. In common with other authors $[17,18]$, we remove the outer edges of cartilage coverage by defining trimmed regions for each joint. Figure 1 illustrates the six regions of interest, trimming boundary, and identifying labels, as shown on the mean bone surfaces. This technique has been described in detail by Williams et al. [19].

\section{Population and difference maps}

The correspondence of the cartilage-thickness maps between scan sessions and across subjects enables us to aggregate the individual subjects' thickness maps to a mean cohort-thickness map for each scanner. Individual maps could be constructed to demonstrate the point-wise differences in thickness between scanner pairs, and these are also combined to form mean cartilage-thickness difference maps for the cohort. These population-difference maps were displayed on the mean bone shape, from which more-localized regional measures could be performed.

\section{Statistical analysis}

The study provided data for the reproducibility analysis of regional cartilage mean thickness between scanners (interscanner) and within the Phillips scanner (intrascanner). Image data from the first scan of the two taken at the Philips site were used only for the interscanner analysis. Intra- and interscanner precision was evaluated with Bland-Altman analysis, paired $t$ tests, and coefficient of variation $(\mathrm{COV})$, aggregated as the root-mean-square over all subjects for scanners pairs and expressed as a percentage [20]. Bland-Altman analysis provides the 'bias' (mean difference) and its limits of agreement between any pairs of measurements [21].

\section{Results}

All data were acquired within a $4 \frac{1}{2}-$-month period. The duration between first and last scans for a single subject was $74 \pm 35$ days; range, 13 to 123 days. Results of the semiquantitative cartilage scoring by WORMS [12] demonstrated a large range of changes within the cohort. Mean cartilage scores for the MTF, LTF, and PF compartments for the cohort were $5.7 \pm 5.9,3.6 \pm 4.7$, and 7.0 \pm 5.1 , respectively (Philips scanner) [22]. Subjects demonstrated a broad range of OA pathology, from mild cartilage abnormalities to full-thickness cartilage loss and subchondral changes.

\section{Intrascanner precision}

The Philips intrascanner COVs based on the two scans were in the range $2.6 \%$ to $4.6 \%$. Low variability is also depicted by the Bland-Altman analysis, which produced mean differences of magnitude $\leq 0.05 \mathrm{~mm}$ and tight $95 \%$ limits of agreement of magnitude $<0.35 \mathrm{~mm}$ and no significant differences between the two scans (paired $t$ test,
$P>0.05)$ for all regions except the MF region, for which the difference was small at $0.05 \mathrm{~mm}$ (Table 2).

\section{Interscanner precision}

For the three scanner pairs, interscanner cartilage volume COVs were on average $2.3 \%$ higher than thickness values for the six regions. Overall, the comparison of the Philips and Siemens scanners gave values similar to those of the Philips intrascanner. The Philips 3D WATSf mean cartilage-thickness measures were $\leq 0.06 \mathrm{~mm}$ thicker across all four main weight-bearing regions (cMF, CLF, LT, and MT) compared with the 3D DESS (Siemens). These small differences were nonsignificant (paired $t$ test, $P>0.05$ ), except for the central medial femur, the result of which can mostly be attributed to an outlier measurement of abnormally thin cartilage in the Siemens scan of one of the subjects.

More pronounced differences in cartilage-thickness measurements were found on the GE scanner compared with the other two vendors' scanners, as is evident in Figure 2. Measures of the mean thickness in the central femur regions were consistently lower on the GE scanner (average bias, $0.13 \mathrm{~mm}$ over the four femoral regions and both scanner pairs) and consistently higher in the tibial compartments (average bias, $0.19 \mathrm{~mm}$ ). Significant interscanner differences were found for several regions, especially when compared with the Philips data $(P<0.05)$.

\section{Thickness-difference maps}

The spatial distribution of differences between scanners is visualized on the mean cartilage-difference thickness maps in Figure 3. The differences are shown by using a consistent red-to-blue scale for all four comparison plots. In general, the largest variability is exhibited at the periphery of the cartilage coverage. The paler-color map for the Philips intrascanner differences reflects the lower variability compared with the interscanner maps. The Philips and Siemens scans showed higher values for femoral cartilage thickness compared with GE, whereas this pattern was reversed in the tibia.

\section{Discussion}

The current interest in multicenter trials using MRimaging biomarkers to measure OA progression [7] requires investigation and optimization of interscanner precision errors. Multicenter trials using 3.0T scanners may require using scanners of different vendors, and therefore acquisition sequences and hardware may differ between sites. This study is the first to compare repeated measures of cartilage morphology from 3.0T MR scanners of three different vendors on symptomatic subjects.

Intrascanner cartilage mean thickness coefficients of variation on the Philips scanner in this study are in the range $2.6 \%$ to $4.6 \%$, which is in line with other intrascanner 
Table 2 Results of Philips intrascanner and vendor interscanner comparisons

\begin{tabular}{|c|c|c|c|c|c|c|}
\hline \multirow[t]{2}{*}{ Scanner pair } & \multirow[t]{2}{*}{ Region } & \multirow[t]{2}{*}{ RMS COV } & \multirow[t]{2}{*}{ Mean difference/bias (mm) } & \multicolumn{2}{|c|}{$95 \%$ limits of agreement $(\mathrm{mm})$} & \multirow{2}{*}{$\begin{array}{l}\text { Paired } t \text { test } \\
P \text { value }\end{array}$} \\
\hline & & & & Lower bound & Upper bound & \\
\hline \multirow[t]{6}{*}{ Philips first to second scan(intrascanner) } & $\mathrm{CLF}$ & $2.6 \%$ & 0.00 & -0.19 & 0.20 & 0.95 \\
\hline & CMF & $3.2 \%$ & 0.03 & -0.14 & 0.20 & 0.23 \\
\hline & LT & $4.1 \%$ & -0.05 & -0.34 & 0.25 & 0.30 \\
\hline & MT & $4.6 \%$ & 0.00 & -0.30 & 0.30 & 0.98 \\
\hline & LF & $3.6 \%$ & 0.00 & -0.23 & 0.23 & 0.98 \\
\hline & MF & $2.8 \%$ & 0.05 & -0.08 & 0.18 & $0.02^{\mathrm{a}}$ \\
\hline \multirow[t]{6}{*}{ Philips to Siemens } & $\mathrm{CLF}$ & $4.1 \%$ & 0.06 & -0.13 & 0.25 & 0.10 \\
\hline & CMF & $5.4 \%$ & 0.05 & -0.19 & 0.30 & $0.04^{\mathrm{a}}$ \\
\hline & LT & $4.0 \%$ & 0.06 & -0.10 & 0.22 & 0.75 \\
\hline & MT & $5.6 \%$ & 0.05 & -0.33 & 0.43 & 0.30 \\
\hline & LF & $4.9 \%$ & 0.13 & -0.04 & 0.30 & $0.00^{\mathrm{a}}$ \\
\hline & MF & $5.8 \%$ & 0.08 & -0.12 & 0.29 & $0.00^{\mathrm{a}}$ \\
\hline \multirow[t]{6}{*}{ Siemens to GE } & $\mathrm{CLF}$ & $5.1 \%$ & 0.12 & -0.11 & 0.36 & $0.00^{\mathrm{a}}$ \\
\hline & $\mathrm{cMF}$ & $5.6 \%$ & 0.13 & -0.06 & 0.31 & $0.00^{\mathrm{a}}$ \\
\hline & $\mathrm{LT}$ & $7.7 \%$ & -0.24 & -0.55 & 0.06 & $0.00^{\mathrm{a}}$ \\
\hline & MT & $7.7 \%$ & -0.17 & -0.51 & 0.18 & $0.01^{\mathrm{a}}$ \\
\hline & LF & $4.2 \%$ & 0.04 & -0.20 & 0.27 & 0.28 \\
\hline & MF & $5.2 \%$ & 0.01 & -0.25 & 0.26 & 0.85 \\
\hline \multirow[t]{6}{*}{ Philips to GE } & $\mathrm{CLF}$ & $6.6 \%$ & 0.18 & -0.03 & 0.39 & $0.00^{\mathrm{a}}$ \\
\hline & $\mathrm{CMF}$ & $9.6 \%$ & 0.21 & -0.03 & 0.46 & $0.00^{\mathrm{a}}$ \\
\hline & LT & $8.1 \%$ & -0.23 & -0.60 & 0.13 & $0.00^{\mathrm{a}}$ \\
\hline & MT & $5.4 \%$ & -0.12 & -0.34 & 0.11 & $0.00^{\mathrm{a}}$ \\
\hline & LF & $7.2 \%$ & 0.17 & -0.14 & 0.47 & $0.00^{\mathrm{a}}$ \\
\hline & MF & $7.3 \%$ & 0.14 & -0.14 & 0.42 & $0.00^{\mathrm{a}}$ \\
\hline
\end{tabular}

${ }^{a} P<0.05$ (between-scanner differences are significant). The Bland-Altman plots (not shown) showed no relations between mean and difference of per-subject measures of regional cartilage thickness. CLF, central lateral femur; CMF, central medial femur; LF, lateral femur; LT, lateral tibia; MF, medial femur; MT, medial tibia; RMS COV, root-mean-square coefficient of variation.

analyses in OA $[23,24]$. Interscanner variability between the Philips and Siemens scanners demonstrated small systematic differences in regional cartilage-thickness measurements that were comparable with the intrascanner precision errors. The results compare favorably with those

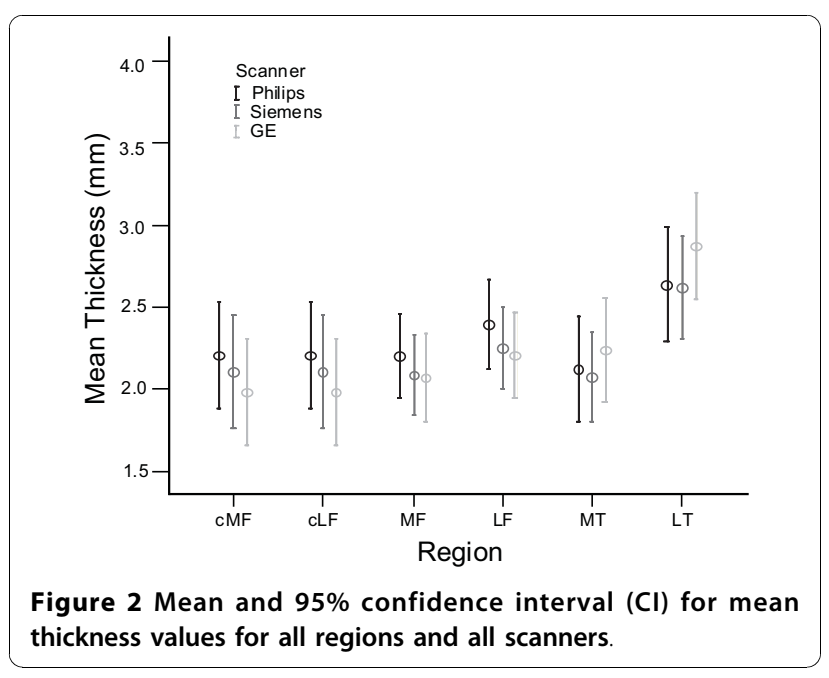

of the previous multivendor study by Morgan et al. [8], which measured knee-cartilage volume in healthy subjects at 1.0 to $1.5 \mathrm{~T}$, where interscanner volume COVs were in the range $9.0 \%$ to $18.7 \%$. This can be explained by the increased SNR, which allowed resolution to be almost doubled in each plane, reducing partial-volume effects. In our study, intrascanner cartilage-volume COVs produced very similar values to the thickness COVs, whereas interscanner volume COVs were on average $2.3 \%$ higher for the given regions. Volume COVs are a function of variability of both thickness and surface area, thus providing limited information on sources of variability in precision errors and therefore not reported in detail here.

The higher interscanner variability measured between Philips and GE can be explained by both hardware and sequence differences. Difficulties were encountered in mimicking the DESS sequence on the GE platform. Increased image noise and differences in sequences (water-excitation on Philips versus fat-saturation on GE) may have an effect on the cartilage boundaries. Comparisons of these sequences have demonstrated apparently thicker cartilage in the water-excitation images, although the differences were in the range of 


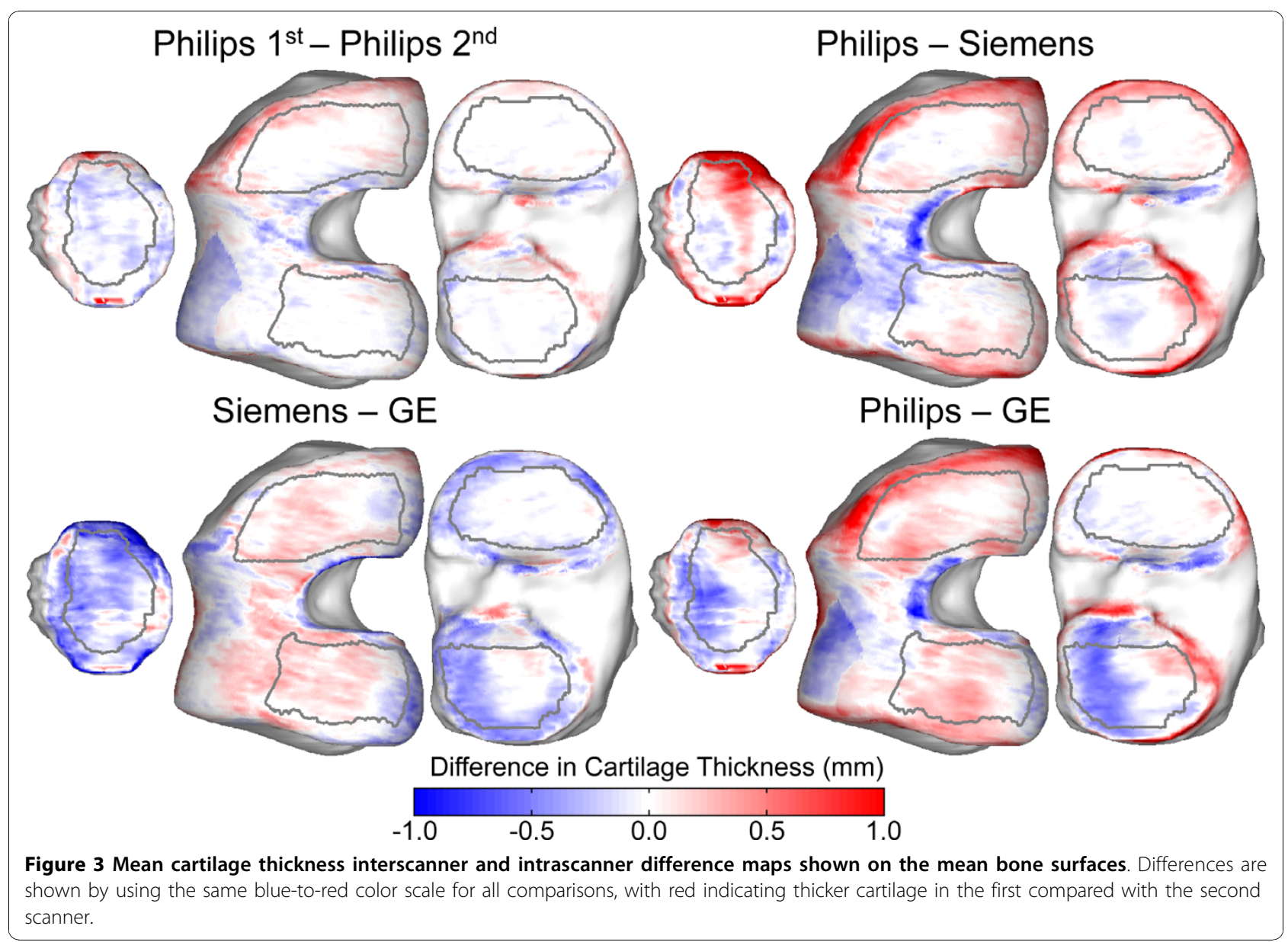

in vivo reproducibility [24]. Although image resolutions were similar, subjectively, the GE images appeared more noisy. Poststudy examination of the images revealed that the cartilage-to-bone boundary was subjectively less pronounced on the GE scanner compared with the Philips scanner and that, when comparing images of the same subjects, in some cases, the femoral cartilage appeared thinner. Partial-volume errors are consequently likely to have been greater. With respect to segmentation of the sagittal 3D sequences; the Philips images were the easiest to segment, believed to be due to postprocessing, which eliminated background noise and accentuated the cartilage edges, aiding the livewire algorithm in segmentation. The Siemens and GE images appeared noisier, although the Siemens DESS sequence provided superior fluid-to-soft tissue contrast at the cartilage surface. Formal assessment of image SNR could not be performed because of the postprocessing of the Philips images. These factors also may account for the underestimation of femoral-cartilage thickness on the GE scanner due to more difficulties encountered delineating the cartilage-to-bone boundary.
A significant variation was found in the QA performed at each site, with formal slice-thickness profile and geometric distortion QA being available at the Philips site only. It is possible that variations in the slice-thickness profile between scanners may have contributed to the interscanner variation because of differences in partialvolume effects. These errors are considered to be most frequent at sites of greater surface curvature at the edges of the cartilage plates, which would have been reduced by the trimming of the segmented cartilage surfaces in the image analysis.

Multivendor studies investigating cartilage morphology have been performed previously by using MR scanners at $3.0 \mathrm{~T}$ and lower field strengths [8,9]. Morgan et al. [8] detected small systematic interscanner differences in quantitative measurements of cartilage volume. Kornaat et al. [9] performed a study with two 3.0T scanners manufactured by Philips and GE. The principal difference of our study is that the subjects are symptomatic. Accuracy and precision of cartilage measurements in thinned cartilage is more challenging because of factors such as partial-volume averaging and altered signal and structure in pathologic tissues. Potential factors affecting variability 
between scanner vendors include differences in hardware, pulse sequences, and so on. At 3.0T, factors such as $B_{1}$ and $B_{0}$ inhomogeneity play a greater role in interscanner variability compared with $1.5 \mathrm{~T}$ [25]. It is important to emphasize that our study compared three specific scanners, and the results cannot necessarily be generalized to all scanners of a particular vendor. We aimed to investigate the likely errors in a multivendor study, not to compare the performances of different vendors' products in general.

Interscanner differences in knee-cartilage thicknesses between Philips and GE 3.0T scanners have been measured by others in healthy subjects [9], where a mean difference of $0.19 \mathrm{~mm}$ (up to a maximum of $0.88 \mathrm{~mm}$ but not statistically significant) in the femoral-cartilage thickness was reported. Similar to this study, the Philips scanner used had an eight-channel phased-array knee RF coil, and the GE scanner had a quadrature transmitreceive coil. Hardware differences are inevitably encountered in other multicenter studies, as often only one RF coil type is available, as was the case in this study.

Minimizing precision errors means that smaller differences can be detected in a group with a given number of subjects. What represents a clinically significant difference in cartilage thickness in OA is still unclear and, we hope, will be answered by the results of the NIH OAI study. This study presents a method intended for monitoring changes in groups of subjects with OA. Our method incorporates a bone-modeling technique that aims to facilitate image segmentation and analysis by achieving anatomic correspondence between images. The model was devised by using data from a training set of images from Siemens scanners. We have shown that this can be successfully applied to similar image data from scanners of different vendors.

\section{Conclusions}

The study demonstrates that the Siemens NIH OAI protocol can be optimized for the Philips and GE 3T scanners to achieve images of similar resolution and contrast. However, in quantitative cartilage-thickness analysis, significant interscanner differences may occur in some cases. These may be due to a combination of differences in extremity RF coil, challenges in sequence optimization, and image postprocessing.

Overall, imaging at 3.0 T improves intra- and interscanner precision errors in comparison with imaging at lower field strengths. This study supports the use of 3.0T scanners from different manufacturers in clinical trials of OA involving quantitative MR cartilage morphology after investigation of interscanner precision errors. It is still recommended, however, that longitudinal observations on individuals be performed on the same scanner.

\begin{abstract}
Abbreviations
COV: coefficient of variation; DESS-we: double echo steady state-water excitation; Fat Sat.: fat saturation; FOV: field of view; LTF: lateral tibiofemoral; MTF: medial tibiofemoral; NIH: National Institutes of Health; OA: osteoarthritis; OAl: osteoarthritis initiative; PF: patellofemoral; SENSE: sensitivity encoding; SNR: signal-to-noise ratio; TE: echo time; TI: inversion time; WATSf: water selective (fluid); WE: water excitation; WORMS: wholeorgan magnetic resonance score.
\end{abstract}

\section{Acknowledgements}

We thank AstraZeneca for funding of MR scans; C. Bos, Philips Medical Systems (Best, Netherlands), for Philips protocol optimization; S. Dezonie, GE Healthcare, UK for GE protocol optimization; G. Green, York Neurolmaging Centre; Lloyd Gregory, Translational Imaging Unit, University of Manchester; and OAI Investigators, OAI Staff, and OAI Coordinating Center.

The OAI is a public-private partnership comprising five contracts (N01-AR-22258; N01-AR-2-2259; N01-AR-2-2260; N01-AR-2-2261;N01-AR-2-2262) funded by the National Institutes of Health, a branch of the Department of Health and Human Services, and conducted by the OAI Study Investigators. Private funding partners include Merck Research Laboratories (Hoddesdon, Herts, UK); Novartis Pharmaceuticals Corporation (Camberley, Surrey, UK); GlaxoSmithKline (Uxbridge, Middlesex, UK); and Pfizer, Inc. (Tadworth, Surrey, UK). Private-sector funding for the OAl is managed by the Foundation for the National Institutes of Health. This manuscript was prepared by using an OAl public-use data set and does not necessarily reflect the opinions or views of the OAI investigators, the $\mathrm{NIH}$, or the private funding partners.

\section{Author details}

'Biomedical Imaging Institute, The University of Manchester, Oxford Road, Manchester, M13 9PT, UK. ${ }^{2}$ Translational sciences, AstraZeneca, Alderley Park, Macclesfield, Cheshire, SK10 4TG, UK. ${ }^{3}$ Imorphics Ltd., Kilburn House, Lloyd Street North, Manchester Science Park, Manchester, M15 6SE, UK. ${ }^{4} \mathrm{NIHR}$ Leeds Musculoskeletal Biomedical Research Unit, Chapel Allerton Hospital, Leeds, LS7 4SA, UK. ${ }^{5}$ Clinical Sciences Research Institute, Clinical Sciences Building, University Hospital-Walsgrave Campus, Clifford Bridge Road, Walsgrave, Coventry, CV2 2DX, UK.

\section{Authors' contributions}

SB is Principal Investigator, and CEH is Chief Investigator for this study. SB, CEH, JCW, and TGW contributed to the conception and design of the study and in the interpretation and discussion in the manuscript. TGW, MB, and CJT contributed to quantitative image analysis. $\mathrm{RH}$ contributed to image sequence optimization and data acquisition. All authors read and approved the final manuscript.

\section{Competing interests}

CJT and MB declare the following financial competing interests: CJT is currently Principal Investigator on a research council grant that involves collaboration with Imorphics, Ltd., continuation of which is dependent on a significant contribution in kind from the company; and $\mathrm{MB}$ is an employee of the company. CJT and MB personally hold shares in Imorphics, Ltd., which is a University spin-out company. Imorphics provides products and services for the quantitative analysis of medical images for clinical trials and holds the commercial exploitation rights for the work described in the article. $\mathrm{SB}, \mathrm{CEH}, \mathrm{JCW}, \mathrm{RH}$, and TGW declare that they have no competing interests.

Received: 11 December 2009 Revised: 14 October 2010 Accepted: 28 October 2010 Published: 28 October 2010

\section{References}

1. The Osteoarthritis Initiative. [http://www.oai.ucsf.edu/datarelease/].

2. Eckstein F, Maschek S, Wirth W, Hudelmaier M, Hitzl W, Wyman B, Nevitt M, Hellio Le Graverand MP: One year change of knee cartilage morphology in the first release of participants from the Osteoarthritis Initiative Progression Subcohort: association with sex, body mass index, symptoms, and radiographic OA status. Ann Rheum Dis 2008, 68:674-679.

3. Eckstein F, Stein V, Lengfelder V, Hudelmaier M, Wirth W, Cahue S, Marshall M, Sharma L: Regional cartilage loss in patients with femorotibial osteoarthritis with neutral, valgus and varus knee alignment. Workshop on imaged-based measures of osteoarthritis Salzburg, Austria; 2007. 
4. Wirth W, Hellio Le Graverand MP, Wyman BT, Maschek S, Hudelmaier M, Hitzl W, Nevitt M, Eckstein F: Regional analysis of femorotibial cartilage loss in a subsample from the Osteoarthritis Initiative progression subcohort. Osteoarthritis Cartilage 2009, 17:291-297.

5. Waterton JC, Solloway S, Foster JE, Keen MC, Gandy S, Middleton BJ, Maciewicz RA, Watt I, Dieppe PA, Taylor CJ: Diurnal variation in the femoral articular cartilage of the knee in young adult humans. Magn Reson Med 2000, 43:126-132.

6. Eckstein $F$, Reiser $M$, Englmeier $\mathrm{KH}$, Putz R: In vivo morphometry and functional analysis of human articular cartilage with quantitative magnetic resonance imaging: from image to data, from data to theory. Anat Embryol (Berl) 2001, 203:147-173.

7. Hellio Le Graverand MP, Wyman B, Buck R, Wirth W, Hudelmaier M, Eckstein F: Twelve month longitudinal change in regional cartilage morphology in a multicenter, multivendor MRI study at 3.0T: the A9001140 Study. Workshop on imaging based biomarkers of osteoarthritis Salzburg, Austria; 2007.

8. Morgan SR, Waterton JC, Maciewicz RA, Leadbetter JE, Gandy SJ, Moots RJ Creamer $P$, Nash AFP: Magnetic resonance imaging measurement of knee cartilage volume in a multicentre study. Rheumatology 2004, 43:19-21.

9. Kornaat PR, Koo S, Andriacchi TP, Bloem JL, Gold GE: Comparison of quantitative cartilage measurements acquired on two 3.0T MRI systems from different manufacturers. J Magn Reson Imaging 2006, 23:770-773.

10. Eckstein F, Buck RJ, Burstein D, Charles HC, Crim J, Hudelmaier M, Hunter DJ, Hutchins G, Jackson C, Kraus VB, Lane NE, Link TM, Majumdar LS, Mazzuca S, Prasad PV, Schnitzer TJ, Taljanovic MS, Vaz A, Wyman B, Le Graverand MP: Precision of 3.0 Tesla quantitative magnetic resonance imaging of cartilage morphology in a multicentre clinical trial. Ann Rheum Dis 2008, 67:1683-1688.

11. Coordinating Center UoCSF: The osteoarthritis initiative: protocol for the cohort study. UoCSF, San Francisco, California; 2006.

12. Peterfy CG, Guermazi A, Zaim S, Tirman PF, Miaux Y, White D, Kothari M, Lu Y, Fye K, Zhao S, Genant HK: Whole-organ magnetic resonance imaging score (WORMS) of the knee in osteoarthritis. Osteoarthritis Cartilage 2004, 12:177-190.

13. Mortensen EN, Barret WA: Interactive segmentation with intelligent scissors. Graph Models Image Process 1998, 60:349-384.

14. Williams TG: Regional quantitative analysis of knee cartilage in a population study using $\{\mathrm{MRI}\}$ and model based correspondences. (ISBI) IEEE international symposium on biomedical imaging Arlington VA; 2006.

15. Petrovic $V$, Cootes TF: Automatic framework for medical image registration, segmentation and modelling (sic). Medical image understanding and analysis Manchester, UK; 2006.

16. Eckstein F, Ateshian G, Burgkart R, Burstein D, Cicuttini F, Dardzinski B, Gray M, Link TM, Majumdar S, Mosher T, Peterfy C, Totterman S, Waterton J, Winalski CS, Felson D: Proposal for a nomenclature for magnetic resonance imaging based measures of articular cartilage in osteoarthritis. Osteoarthritis Cartilage 2006, 14:974-983.

17. Tamez-Pena JG, Barbu-McInnis M, Totterman S: Unsupervised definition of the tibia-femoral joint regions of the human knee and its applications tocartilage analysis. Medical Imaging: Image Processing Proc of SPIE 2006, $6144,61444 \mathrm{~K}$

18. Wirth E, Roth M: Regional analysis of cartilage morphology in defined anatomical subregions of femorotibial cartilages. OARSI World Congress on Osteoarthritis Prague, Czech Republic; 2006.

19. Williams TG, Holmes AP, Bowes M, Vincent G, Hutchinson CE, Waterton JC, Maciewicz RA, Taylor CJ: Measurement and visualisation of focal cartilage thickness change from MRI in a study of knee osteoarthritis using a novel image analysis tool. Br J Radiol 2010, 83:940-948.

20. Gandy SJ, Dieppe PA, Keen MC, Maciewicz RA, Watt I, Waterton JC: No loss of cartilage volume over three years in patients with knee osteoarthritis as assessed by magnetic resonance imaging. Osteoarthritis Cartilage 2002, 10:929-937.

21. Bland JM, Altman DG: Statistical methods for assessing agreement between two methods of clinical measurement. Lancet 1986, 1:307-310.

22. Balamoody S: MR imaging at 3.0T in the assessment of knee osteoarthritis. University of Manchester, Imaging Sciences and Biomedical Engineering 2008, MD Thesis.

23. Eckstein F, Charles HC, Buck RJ, Kraus VB, Remmers AE, Hudelmaier M, Wirth W, Evelhoch JL: Accuracy and precision of quantitative assessment of cartilage morphology by magnetic resonance imaging at 3.0T. Arthritis Rheum 2005, 52:3132-3136.

24. Glaser C, Burgkart R, Kutschera A, Englmeier KH, Reiser M, Eckstein F: Femoro-tibial cartilage metrics from coronal MR image data: technique, test-retest reproducibility, and findings in osteoarthritis. Magn Reson Med 2003, 50:1229-1236.

25. Multi-centre studies: how to reduce inter-centre differences. [http://qmri. org/].

doi:10.1186/ar3174

Cite this article as: Balamoody et al:: Comparison of 3T MR scanners in regional cartilage-thickness analysis in osteoarthritis: a cross-sectional multicenter, multivendor study. Arthritis Research \& Therapy 2010 12:R202.

\section{Submit your next manuscript to BioMed Central and take full advantage of:}

- Convenient online submission

- Thorough peer review

- No space constraints or color figure charges

- Immediate publication on acceptance

- Inclusion in PubMed, CAS, Scopus and Google Scholar

- Research which is freely available for redistribution

Submit your manuscript at www.biomedcentral.com/submit
Biomed Central 